\title{
Erratum
}

\section{Erratum to: Perturbation Formulas for Traces on Normed Ideals}

\author{
Ken Dykema ${ }^{1}$, Anna Skripka ${ }^{2}$ \\ ${ }^{1}$ Department of Mathematics, Texas A\&M University, College Station, TX 77843-3368, USA. \\ E-mail: kdykema@math.tamu.edu \\ 2 Department of Mathematics and Statistics, University of New Mexico, 400 Yale Blvd NE, \\ MSC01 1115, Albuquerque, NM 87131, USA. E-mail: skripka@math.unm.edu
}

Received: 18 May 2015 / Accepted: 19 June 2015

Published online: 16 July 2015 - @ S Springer-Verlag Berlin Heidelberg 2015

Commun. Math. Phys. 325, 1107-1138 (2014)

In the case of a type $\mathrm{II}_{\infty}$ factor, the definition of $\|A\|_{\mathcal{I}}$ found in Eq. (2.4) of the original article should be modified to

$$
\mathcal{I}=\left\{A \in \mathcal{B} \mid\|A\|_{\mathcal{I}}:=\max \left\{\|A\|, \sup _{t>0} \frac{1}{\psi(t)} \int_{0}^{t} \mu_{s}(A) d s<\infty\right\}\right\},
$$

where $\|\cdot\|$ is the operator norm. We note that Proposition 2.5 holds also with this new definition of $\|\cdot\|_{\mathcal{I}}$, making use of the existing proof for the case of the old definition of $\|\cdot\|_{\mathcal{I}}$ and the fact that for the operator norm, we have $\|B\|=\||B|\|=\left\||B|^{p} \mid\right\|^{1 / p}$. This modification is needed so that condition (ii) of Definition 2.1 (of an ideal norm) holds. As this condition holds automatically for Marcinkiewicz ideals in type $\mathrm{I}_{\infty}$ factors, the unmodified form of Eq. (2.4) may be used for $\mathrm{I}_{\infty}$ factors.

Communicated by H.-T. Yau 